\title{
Biotechnology and modifications of the rumen microbial ecosystem
}

\section{By Robert B. Hespell, Northern Regional Research Center, Agricultural Research Service, USDA, 1815 North University Street, Peoria, Illinois 61604, USA}

The exploitation of rumen functions by manipulation of the rumen micro-organisms is not a new concept. For several decades, researchers in universities and private companies have been trying to develop various methods to chemically treat specific feedstuffs in order to modify their susceptibilities to digestion in the rumen, or to develop compounds that modify the rumen fermentation pattern by affecting the growth or metabolism of various rumen micro-organisms. Relative to the considerable amount of work put into these sort of research areas, the levels of success have been rather minimal. This low success rate is partly due to both the lack of knowledge and the underestimation of the complexities in the physicochemical architecture of plant materials and of the rumen microbial ecosystem. With the rapid new developments in molecular biology, recombinant-DNA technology, and computer-based instrumentation over the last decade, there has been a renewed interest towards manipulation of the rumen fermentative processes via use of genetically engineered micro-organisms, particularly with species of rumen origin. Assuming that the large gaps in our knowledge on the genetic and biochemical aspects of these micro-organisms can be adequately fulfilled by research in the next few years, it is reasonable to expect that genetically engineered rumen micro-organisms will be available and will possibly be in commercial use in the next decade.

The idea of using genetically engineered micro-organisms in the rumen immediately conjures up a number of questions in the minds of many persons. Some of these questions can be and need to be answered immediately. First, given the co-evolution of the ruminant animal and its microbes over the last 60-70 million years, wouldn't one expect via natural selection that the 'best' micro-organism strains are already present in the animal? The answer, simply stated, is no! The superior farm animals that exist today did not arise from pure natural selection, but from highly intensive, man-made breeding and feeding programmes. There is no substantial reason to doubt that selection for improved 'stocks' of micro-organisms can be done and, most probably, many of the microbial strains in domesticated animals have some superior characteristics over those strains found in wild animals such as deer, caribou or kangaroos. That rumen microbial populations and gut digestive systems of domestic animals are still less than satisfactory is readily evident from the levels of undegraded feedstuffs present in voided faecal material, particularly starch in high-grain-level-fed beef animals in intensive feedlot farming operations. A second question that almost invariably arises is 'what type of super micro-organism would be created to increase feedstuff digestion, microbial cell protein levels, and volatile fatty acid production to increase animal performance? Because of the wide range of feedstuffs used, the varying complexities in the physicochemical structures of plant cell materials, and the myriad interactions within the rumen microbial populations, it would seem almost impossible to genetically engineer a microbial species into a super micro-organism capable of rapidly digesting all sorts of feedstuffs given to different ruminants under various feeding and grazing regimens. Similarly, at the animal level, genetic selection has not, and most likely will not, produce a super breed having all the desired growth and reproductive traits. On the other hand, recombinant-DNA technology can provide the means to rapidly and selectively modify specific genes or 
Table 1. Major bacterial species of the rumen and their biochemical activities

\begin{tabular}{lll}
\multicolumn{1}{c}{$\begin{array}{c}\text { Species } \\
\text { Ruminococcus albus }\end{array}$} & $\begin{array}{c}\text { Rumen } \\
\text { function* }\end{array}$ & $\begin{array}{c}\text { Fermentation } \\
\text { products }\end{array}$ \\
Ruminococcus flavefaciens & $\mathrm{C}, \mathrm{X}$ & $\mathrm{F}, \mathrm{A}, \mathrm{E}$ \\
Butyrivibrio fibrisolvens & $\mathrm{C}, \mathrm{X}$ & $\mathrm{F}, \mathrm{S}$ \\
Bacteroides succinogenes & $\mathrm{C}, \mathrm{X}, \mathrm{PR}, \mathrm{P}, \mathrm{A}, \mathrm{L}, \mathrm{SU}, \mathrm{AU}$ & $\mathrm{F}, \mathrm{A}, \mathrm{B}, \mathrm{L}$ \\
Bacteroides amylophilus & $\mathrm{C}$ & $\mathrm{F}, \mathrm{S}$ \\
Bacteroides ruminicola & $\mathrm{A}, \mathrm{PR}$ & $\mathrm{F}, \mathrm{A}, \mathrm{S}$ \\
Selenomonas ruminantium & $\mathrm{A}, \mathrm{X}, \mathrm{P}, \mathrm{PR}, \mathrm{SU}$ & $\mathrm{F}, \mathrm{A}, \mathrm{S}$ \\
Anaerovibrio lipolytica & $\mathrm{A}, \mathrm{SU}, \mathrm{GU}, \mathrm{LU}, \mathrm{LP}$ & $\mathrm{F}, \mathrm{A}, \mathrm{L}, \mathrm{P}$ \\
Lachnospira multiparus & $\mathrm{L}, \mathrm{GU}$ & $\mathrm{A}, \mathrm{P}, \mathrm{S}$ \\
Succinivibrio dextrinosolvens & $\mathrm{P}, \mathrm{A}, \mathrm{PR}$ & $\mathrm{F}, \mathrm{A}, \mathrm{L}, \mathrm{E}$ \\
Streptococcus bovis & $\mathrm{D}, \mathrm{P}$ & $\mathrm{F}, \mathrm{A}, \mathrm{S}$ \\
Wolinella succinogenes & $\mathrm{A}, \mathrm{SU}, \mathrm{PR}(?), \mathrm{LP}$ & $\mathrm{F}, \mathrm{A}, \mathrm{L}$ \\
Methanobrevibacter ruminantium & $\mathrm{HU}$ & $\mathrm{S}$ \\
Methanosarcina barkeri & $\mathrm{HU}, \mathrm{M}$ & $\mathrm{M}$ \\
& $\mathrm{HU}, \mathrm{AU}, \mathrm{M}$ & $\mathrm{M}$
\end{tabular}

${ }^{*} \mathrm{C}$, cellulolytic; X, xylanolytic; P, pectinolytic; A, amylolytic; D, dextrinolytic; L, lipolytic; PR, proteolytic; SU, soluble-sugar user; GU, glycerol user; LU, lactate user; HU, hydrogen user; AU, acetate user; LP, lactogenic; $M$, methanogenic.

$\dagger F$, formate; A, acetate; $P$, propionate; L, lactate; S, succinate; B, butyrate; E, ethanol; $M$, methane.

introduce new genes into a species, particularly at the microbial level, that endow the species with specific traits. Some of the types of genetic engineering that may be possible with rumen bacteria and the limitations towards attaining these goals are discussed later.

\section{Some microbial and ecological considerations}

The rumen contains a complex microbial ecosystem comprising mainly strictly anaerobic bacteria and protozoa with small numbers of anaerobic fungi and facultatively anaerobic bacteria. Biochemical and genetic studies must be limited to using species of the anaerobic bacteria and fungi because the protozoa cannot be readily grown in pure cultures. The aerobes and the facultative anaerobes constitute numerically insignificant populations that contribute little to the overall microbial activities in the rumen. The dense bacterial population $\left(10^{10}-10^{11} \mathrm{cel} / \mathrm{ml}\right)$ in all probability contains several hundred species, but only about thirty species are significant in numbers $\left(10^{7} \mathrm{cells} / \mathrm{ml}\right.$ or greater $)$, in biochemical activities, and in distribution in various ruminants of diverse geographic location. These major bacterial species have been well characterized with respect to their morphologies, growth characteristics, and other phenotypic traits as well as their functional roles within the rumen ecosystem (Table 1). Thus, it is possible to select specific rumen bacterial species having biochemical activities (e.g. starch degradation) which are well expressed in the ecosystem and are possible targets for genetic engineering.

A wide variety of ecological factors are involved in determining whether or not a bacterial species can exist and grow within the rumen. Although the number and types of these ecological factors are not completely known and may vary from species to species, the numerous studies with pure cultures of rumen bacteria have suggested some of the more important microbial ones. These include: (a) ability to attach to feed particles or to associate with the adherent cell populations, or both; (b) tolerance to high levels of volatile fatty acids (about 100-150 mM) and high-salt conditions (about 50-60 g sodium chloride/kg); (c) high affinities for soluble mono- and oligomeric forms of carbohydrates 
and amino acids; (d) rather low expenditures of energy for maintenance functions during growth; (e) generally high growth yields with respect to energy formed. It is thought that these microbial traits along with rumen environmental factors are important in precluding facultative anaerobes, soil-borne and other non-rumen micro-organisms from establishing any numerically significant populations in the rumen. Thus, it is rather improbable that a genetically engineered strain of well-studied bacteria such as Escherichia coli, or species of Bacillus, Staphylococcus or Streptococcus will exist in the rumen. In addition, any genetic engineering of a rumen bacterial species, such as amplification of an amylase gene, must not dramatically affect the organism's ecological traits that allow it to exist in the rumen.

\section{Modifications of rumen functions}

Although much information is known about the rumen ecosystem, there are large gaps in our knowledge regarding aspects of animal physiology (e.g. regulation of feed intake, salivation, rumen motility) and in microbial physiology (e.g. regulation and biochemical properties of extracellular plant cell wall hydrolytic enzymes, of assimilation of nitrogen compounds, of in situ metabolic cross-feeding between microbial species, or of adherence of micro-organisms to plant materials). These gaps in our knowledge, coupled with the complexities of a twenty to thirty species-dominated ecosystem, preclude treating the rumen simply as a large fermentation vat that is similar to a commercial fermentation process which produces beer, wine, vinegar or organic acids. The possible use of genetically engineered bacterial species in a given commercial process carries with it a rather good probability of success due, in a large part, to the abilities to control the process in terms of nutrient flows, $\mathrm{pH}$, temperature and a variety of other factors. Similar controls are not readily available to rumen fermentation, but can be approached in certain animal farming situations. For example, farm operations where the animals are under high degrees of management, the types of feedstuffs used are well defined, and feed intake is monitored or controlled or both, have an increased probability for controlling the rumen ecosystem. Such farming operations would include the modern automated dairy farms, beef feedlot systems, and situations where animals are being given defined diets before and during shipment. These farming operations are better suited for the introduction of genetically engineered micro-organisms, and for the maintenance over time of the introduced species. In contrast, animal farming operations involving low management input or use of grazing animals would seem to be less amenable to the use of genetically engineered rumen micro-organisms because of their increased inherent variables and, possibly, because of economic factors.

One area for manipulation of rumen fermentation via use of genetically engineered micro-organisms would be to focus on changing the rumen digestion of specific dietary components. Numerous animal digestion studies have shown that rumen starch digestion is somewhat incomplete, particularly with high-grain-level-fed beef animals having high feed intakes. In this case, introduction of a genetically engineered bacterial species having high expression of amylases for starch degradation and, possibly, high xylanase (EC 3.2.1.32) activities for bran-hemicellulose degradation, would seem feasible. In contrast, rumen digestion of dietary proteins often occurs at a greater rate than that for polysaccharides. This often results in rumen ammonia levels that are in excess of microbial and animal needs and loss of dietary $\mathrm{N}$ from the animal through urinary excretion. Based on current knowledge, protease production by rumen bacterial species appears to be constitutive. Genetically then, the problem of excessive proteolysis might be approached by linking the protease gene(s) of one or more species to other $\mathrm{N}$ metabolism genes whose expression is regulated by ammonia levels. Such a gene would 
be urease (EC 3.5.1.5) or glutamine synthetase (EC 6.3.1.2) which have been studied in rumen micro-organism species such as Selenomonas ruminantium (Smith et al. 1981) and Succinivibrio dextrinosolvens (Patterson \& Hespell, 1985). Overall plant fibre digestion in the rumen is commonly slow and incomplete with most animals, particularly when feed intake levels are high. Fibre digestion might be improved by amplifying the cellulolytic and related activities of bacterial species such as Ruminococcus albus, $R$. flavefaciens, Bacteroides succinogenes or Butyrivibrio fibrisolvens. However, cellulolytic activities are usually associated with large complexes of a number of proteins (Stack \& Hungate, 1984) and amplification may require cloning or modifying a large number of genes, or both, along with possibly altering these acid-sensitive species to be more tolerant of lower rumen $\mathrm{pH}$ conditions. Furthermore, increased cellulolysis with animals fed on high levels of grain, lush legumes or citrus pulps (i.e. starch and pectin) would not seem particularly advantageous.

The regulation of fermentation products made by the rumen microbial population would be another target area for use of genetically engineered micro-organisms. Ideally, one would like to balance the arrays and concentrations of fermentation acids to the particular needs of the animal. For steers, this would be to increase propionate usually at the expense of acetate. This might be done by amplifying the genes for selected enzymes involved with propionate formation or increasing the rumen numbers of propionate-forming bacteria such as $S$. ruminantium, Bacteroides ruminicola, Megasphera elsdenii or Veionella species. Because of the limited types of fermentation acids made and the wide number of species producing them in the rumen, directed changes along these lines by simple genetic engineering approaches will be difficult. However, the high lactate production found with shifts to and during feeding of high levels of grains or lush legumes to animals is an overall negative effect. Lactate acidosis could be reduced or brought under control by increasing the abilities of certain bacteria to utilize lactate. $S$. ruminantium has a rather poor affinity for lactate (Russell \& Baldwin, 1978) and genetic modifications of the appropriate binding proteins or transport mechanisms for lactate might be the only genetic engineering needed to turn this species into a super-lactateusing organism.

A third area for manipulation of rumen fermentation would be control of the numbers of specific microbial species in the rumen population. An approach to this problem would be to genetically engineer a bacterial species to produce and secrete a compound that would inhibit the growth or be lethal to another micro-organism. A variation of this approach would not have the bacterial species make the compound, but simply to produce an enzyme that transforms the compound from a latent form to an active form. The latent form would be provided by additions to the feed materials, which also could serve as a means of regulating the overall process. An alternative regulation mechanism could be to link the genetically introduced genes to the inducible expression of other genes (e.g. amylase) in the genetically modified species. The control of the rumen protozoal population by inhibition compounds would seem attractive because their eukaryotic cell nature would allow them to be susceptible to a number of compounds that would have little or no effect on the prokaryotic bacterial cells. However, the rumen methanogenic micro-organisms could also be sensitive because of their archaebacterial cell nature (Kandler \& Konig, 1985) and loss of these hydrogen-gas-utilizing methanogenic organisms would drastically disrupt the entire rumen fermentation system. The metabolism of other bacterial species would also have to be genetically engineered to provide a hydrogen sink. One possibility would be to engineer Eubacterium limosum, a relatively numerically minor species in the rumen, to preferentially form acetate and butyrate from $\mathrm{H}_{2}$ and carbon dioxide. Elimination of rumen methane production is 
Table 2. Research requirements for genetic engineering of rumen bacteria

(1) Genetics research

Identifying genes for selection of recombinant cells.

Cloning of DNA fragments; vectors or vehicles.

DNA delivery systems: transformation, conjugation.

Gene expression: regulatory (promoter sites; ribosomal binding); amplification (copy number; gene fusions).

(2) Biochemical research

Enzymology of enzyme complexes:

Number and types of enzymes involved.

Purification of enzymes.

Properties: substrate specificities, molecular weights, amino acid sequences, physicochemical aspects.

Formation of enzyme complexes:

Secretory apparatus: mechanisms, proteins.

Structural/catalytic proteins: types, ratios.

Involvement of extracellular polysaccharides.

(3) Physiology research

Growth aspects: parental $v$. modified strains.

Quantification of cell numbers and activities.

Stabilities of genetic traits.

Competition and ecological factors: suitable growth or activities in rumen.

attractive since it represents a loss of 6-10\% of the digestible feed energy. Finally, by controlling the numbers of Streptococcus bovis and other rapidly growing, lactateproducing bacterial species, the problem of lactate acidosis may also be eliminated or reduced.

\section{Research requirements}

At the current time, there is insufficient knowledge on rumen bacteria to accomplish manipulations of rumen fermentation by genetic engineering. Simply stated, knowledge about the genetics of rumen bacteria is non-existent. While for a few species some biochemical information on intracellular enzymes related to fermentative energy metabolism and to $\mathbf{N}$ metabolism is available, almost nothing is known about the biochemical properties of extracellular or cell-surface-bound enzymes such as amylases, cellulases, xylanases, pectinases or other enzymes for degradation of plant cell components. Similarly, very little is known about the effects of growth rate, $\mathrm{pH}$, temperature, induction-repression mechanisms or other physiological factors on the expression of these degradative enzymes. As has been discussed in detail (Smith \& Hespell, 1983), concomitant research in these areas (Table 2) must be undertaken in order to successfully change any one rumen bacterial species by genetic engineering.

In the area of genetics research, at least three aspects need to be addressed. First, finding genes that are suitable for selecting and identifying strains that have acquired or lost genetic material. These marker strains might be developed by mutagenic techniques using u.v. light or chemical agents (nitrous acid, nitrosoguanidine) with rumen species to generate mutants auxotrophic for specific amino acids or sugars. A second aspect of genetic research has to include finding or making suitable vehicles to carry the genes of interest and to allow for gene replication or expression independent of chromosomally located genes, or both. It would be highly preferable for this vehicle to be a 
shuttle-vector plasmid capable of replication in the rumen species and in a well-studied bacterium (e.g. Escherichia coli or Bacillus subtilis). The genes or DNA fragments of the rumen species cloned into such a vector can then be subjected to the wide range of genetic tools and analyses available with these well-studied organisms. Finally and most importantly, one must develop a system(s) for introducing DNA into the cells of the rumen bacterium under study. Probably the best system may be transformation as there are a number of available techniques used with other bacteria. However, transfer of DNA by conjugation methods has been successfully used with anaerobes of human colonic origin (Smith et al. 1982).

With respect to biochemical aspects, a variety of areas need to be researched. The types and numbers of enzymes involved in degradation of a given feed component, say xylans or plant proteins, have to be determined along with molecular weights, amino acid sequences, mechanisms and specificities of substrate degradation, $\mathrm{pH}$ and temperature stabilities, etc. This information will be necessary for the development of specific, reliable biochemical methods (a) for ascertaining whether the proteins made by cloned genes are identical to those expressed by the parental strains; (b) for screening for transformed or recombinant cells; or (c) for determining which enzymes or proteins of enzyme complexes need to be amplified or controlled to attain the desired biochemical activity. A clear understanding of the mechanisms associated with the excretion of enzymes to the extracellular environment in rumen bacteria is needed, and may be very important towards obtaining successful gene expression in a non-rumen species having genes cloned from a rumen bacterium.

Once a rumen species has been genetically modified, the impact of this engineering on the general physiology of the strain must be assessed. The overproduction of one or more proteins may result in altered nutrient requirements, growth rates or cell-surface properties. These possible changes need to be determined to see whether the genetically modified strain can grow and effectively compete in mixed microbial populations as found in the rumen. A variety of in vitro studies using cultures of mixed rumen bacteria and the genetically modified species growing on appropriate feedstuff materials will be needed to evaluate the efficacies of various strains or types of the modified species. Both alterations in feed digestion patterns and relative genetic stabilities of the genetically modified species should be monitored under these pseudo-rumen conditions to select the best strain for future testing with animals.

\section{Future prospects}

Alterations in the rumen microbial population and rumen digestion of feedstuffs by use of genetically engineered rumen microbes hold reasonable promise for the future. Much research needs to be done on the genetics and biochemistry of these organisms before success will be realized. This research, however, should contribute significantly to clarifying our understanding of regulatory mechanisms and feedstuff digestion. Overall, such new information will aid in devising new means of treating feedstuffs or use of new dietary components, or both, to improve rumen digestion per se, and allow effective use of genetically engineered strains in the rumen.

\section{REFERENCES}

Kandler, O. \& Konig, H. (1985). In The Bacteria, vol. 8, Archaebacteria, pp. 413-458 [C. R. Woese and R. S. Wolfe, editors). Orlando, Florida: Academic Press.

Patterson, J. A. \& Hespell, R. B. (1985). Applied and Environmental Microbiology 50, 1014-1020.

Russell, J. B. \& Baldwin, R. L. (1978). Applied and Environmental Microbiology 37, 531-536. 
Smith, C. J. \& Hespell, R. B. (1983). Journal of Dairy Science 66, 1536-1546.

Smith, C. J., Hespell, R. B. \& Bryant, M. P. (1981). Applied and Environmental Microbiology 42, 89-96.

Smith, C. J., Welch, R. A. \& Macrina, F. L. (1982). Journal of Bacteriology 151, 281-287.

Stack, R. J. \& Hungate, R. E. (1984). Applied and Environmental Microbiology 48, 218-223. 